\title{
APPLICATIONS OF IYENGAR'S TYPE INEQUALITIES TO.THE ESTIMATION OF ERROR BOUNDS FOR THE TRAPEZOIDAL QUADRATURE RULE*
}

\author{
SEVER S. DRAGOMIR AND SONG WANG
}

\begin{abstract}
In this paper we discuss some applications of the classical lyengar's inequality and its generalization by Agarwal and Dragomir [1] to the estimation of error bounds for the trapezoidal quadrature rule in numerical integration.
\end{abstract}

\section{Introduction}

In 1938 Iyengar proved the following theorem (cf., for example, [3,p.471]);

Theorem 1.1. Let $f$ be a differentiable function on $(a, b)$ and assume that there is a constant $M>0$ such that $\left|f^{\prime}(x)\right| \leq M, \forall x \in(a, b)$. Then we have

$$
\left|\int_{a}^{b} f(x) d x-(b-a) \frac{f(a)+f(b)}{2}\right| \leq \frac{M(b-a)^{2}}{4}-\frac{1}{4 M}(f(b)-f(a))^{2} .
$$

Using the classical inequality due to Hayashi (cf., for example, [2, pp. 311-312]), Agarwal and Dragomir proved in [1] the following generalization of Theorem 1.1.

Theorem 1.2. Let $f: I \subseteq \mathbb{R} \mapsto \mathbb{R}$ be a differentiable mapping in $\dot{I}$, the interior of $I$, and let $a, b \in \dot{I}$ with $a<b$. Let $M=\sup _{x \in[a, b]} f^{\prime}(x)<\infty$ and $m=\inf _{x \in[a, b]} f^{\prime}(x)>$ $-\infty$. If $m<M$ and $f^{\prime}$ is integrable on $[a, b]$, then we have

$$
\begin{aligned}
\left|\frac{1}{b-a} \int_{a}^{b} f(t) d t-\frac{f(a)+f(b)}{2}\right| & \leq \frac{[f(b)-f(a)-m(b-a)][M(b-a)-f(b)+f(a)]}{2(M-m)(b-a)} \\
& \leq \frac{(M-m)(b-a)}{8}
\end{aligned}
$$

Note that if we put $m=-M$ in (1.2). then we get Iyengar's result (1.1).

Applications of (1.2) to some convex mappings and to some special means can be found in [1]. In this paper we apply (1.2) to the estimation of error bounds for the classical trapezoidal quadrature rule in numerical integration.

2. Applications to the trapezoidal quadrature rule

We first discuss the application of Theorem 1.1 to the error estimation for the classical trapezoidal quadrature rule.

Received May 6, 1997.

"This paper is partially supported by a research grant from the Australian Reserch Council 
Theorem 2.1 Let $f:(a, b) \subseteq \mathbb{R} \mapsto \mathbb{R}$ be a differentiable function on $(a, b)$ and assume that $f^{\prime}$ is integrable on $(a, b)$ and $0<\left\|f^{\prime}\right\|_{\infty}:=\sup _{x \in(a, b)}\left|f^{\prime}(x)\right|<\infty$. Let $I_{h}: a=x_{0}<x_{1}<\cdots<x_{n-1}<x_{n}=b$ be a partitioning of $(a, b)$. Then we have

$$
\begin{aligned}
\left|\int_{a}^{b} f(x) d x-T\left(f, I_{h}\right)\right| & \leq \frac{\left\|f^{\prime}\right\|_{\infty}}{4} \sum_{i=0}^{n-1} h_{i}^{2}-\frac{1}{4\left\|f^{\prime}\right\|_{\infty}} \sum_{i=0}^{n-1}\left(f\left(x_{i+1}\right)-f\left(x_{i}\right)\right)^{2} \\
& \leq \frac{\left\|f^{\prime}\right\|_{\infty}}{4} \sum_{i=0}^{n-1} h_{i}^{2}-\frac{1}{4 n\left\|f^{\prime}\right\|_{\infty}}(f(b)-f(a))^{2} \\
& \leq \frac{\left\|f^{\prime}\right\|_{\infty}}{4} \sum_{i=0}^{n-1} h_{i}^{2}
\end{aligned}
$$

where

$$
T\left(f, I_{h}\right):=\sum_{i=0}^{n-1}\left[\frac{f\left(x_{i}\right)+f\left(x_{i+1}\right)}{2}\right] h_{i}
$$

and $h_{i}:=x_{i+1}-x_{i}$ for all $i=0,1, \cdots, n-1$.

Proof. Applying (1.1) to $f(x)$ on $\left[x_{i}, x_{i+1}\right]$ we get

$$
\left|\int_{x_{i}}^{x_{i+1}} f(x) d x-\frac{f\left(x_{i}\right)+f(x i+1)}{2} h_{i}\right| \leq \frac{\left\|f^{\prime}\right\|_{\infty} h_{i}^{2}}{4}-\frac{\left(f\left(x_{i+1}\right)-f\left(x_{i}\right)\right)^{2}}{4\left\|f^{\prime}\right\|_{\infty}}
$$

for all $i=0,1, \cdots, n-1$. Summing over $\mathrm{i}$ and using the triangle inequality we obtain

$$
\left|\int_{a}^{b} f(x) d x-t\left(f, I_{h}\right)\right| \leq \frac{\left\|f^{\prime}\right\|_{\infty}}{4} \sum_{i=0}^{n-1} h_{i}^{2}-\frac{1}{4\left\|f^{\prime}\right\|_{\infty}} \sum_{i=0}^{n-1}\left(f\left(x_{i+1}\right)-f\left(x_{i}\right)\right)^{2}
$$

and thus the first inequality in (2.1) is proved.

Now, using Cauchy-Schwarz inequality we have

$$
n \sum_{i=0}^{n-1}\left(f\left(x_{i+1}\right)-f\left(x_{i}\right)\right)^{2} \geq\left[\sum_{i=0}^{n-1}\left(f\left(x_{i}+1\right)-f(x)\right)\right]^{2}=(f(b)-f(a))^{2}
$$

and thus

$$
-\sum_{i=0}^{n-1}\left(f\left(x_{i+1}\right)-f\left(x_{i}\right)\right)^{2} \leq-\frac{1}{n}(f(b)-f(a))^{2}
$$

Combining this and the first part of (2.1) we have the second inequality in (2.1).

The last inequality in (2.1) is obvious and thus we have proved the theorcm.

Let $I_{n}$ denote the equidistance partitioning of $[a, b]$ given by

$$
I_{n}: x_{i}=a+\frac{b-a}{n} i, i=0,1, \cdots, n \text {. }
$$

For this partitioning we have the following corollary. 
Corollary 2.2. With the assumptions in Theorem 2.1, we have

$$
\left|\int_{a}^{b} f(x) d x-T\left(f, I_{n}\right)\right| \leq \frac{\left\|f^{\prime}\right\|_{\infty}^{2}(b-a)^{2}-(f(b)-f(a))^{2}}{4 n\left\|f^{\prime}\right\|_{\infty}} \leq \frac{(b-a)^{2}\left\|f^{\prime}\right\|_{\infty}}{4 n}
$$

where $T$ is the trapezoidal quadrature rule defined in Theorem 2.1 and $I_{n}$ is the equidistance partitioning defined in (2.2).

Given any $\varepsilon>0$, using (2.3) we are able to give the minimum number of nodes such that the error in the numerical integration based on the equi-distance trapezoidal rule is smaller than $\varepsilon$. This is contained in the following corollary:

Corollary 2.3. Given any constant $\varepsilon>0$, if

$$
n \geq\left[\frac{\left\|f^{\prime}\right\|_{\infty}(b-a)^{2}-(f(b)-f(a))^{2}}{4 \varepsilon\left\|f^{\prime}\right\|_{\infty}}\right]+1
$$

then we have

$$
\left|\int_{a}^{b} f(x) d x-T\left(f, I_{n}\right)\right| \leq \varepsilon
$$

where [u] denotes the integer part of $u$ for any $u \in \mathbb{R}$.

We now consider the application of Theorem 1.2 to the trapezoidal quadrature rule, which is established in the following theorem.

Theorem 2.5. Let $f$ be a differentiable function on $[a, b]$ with $f^{\prime}$ integrable. Let $M:=$ $\sup _{x \in[a, b]} f^{\prime}(x)<\infty$ and $m:=\inf _{x \in[a, b]} f^{\prime}(x)>-\infty$. If $M>m$ then we have

$$
\begin{aligned}
\left|\int_{a}^{b} f(x) d x-T\left(f, I_{h}\right)\right| & \leq \frac{1}{2(M-m)} \sum_{i=0}^{n-1}\left[f\left(x_{i+1}\right)-f\left(x_{i}\right)-m h_{i}\right]\left[M h_{i}-f\left(x_{i+1}\right)+f\left(x_{i}\right)\right] \\
& \leq \frac{M-m}{8} \sum_{i=0}^{n-1} h_{i}^{2}
\end{aligned}
$$

where $I_{h}, T\left(f, I_{h}\right)$ and $h_{i}$ are defined in Theorem 2.1.

Proof. Applying (1.2) to $f$ on the interval $\left[x_{i}, x_{i+1}\right]$ we have

$$
\begin{aligned}
\left|\int_{x_{i}}^{x_{i+1}} f(x) d x-\frac{f\left(x_{i}\right)+f\left(x_{i+1}\right)}{2} h_{i}\right| \leq & \frac{1}{2(M-m)}\left[f\left(x_{i+1}\right)-f\left(x_{i}\right)-m h i\right] \\
& \cdot\left[M h_{i}-f\left(x_{i+1}\right)+f\left(x_{i}\right)\right] \\
\leq & \frac{(M-m) h_{i}^{2}}{8}
\end{aligned}
$$

for all $i=0,1, \cdots, n-1$. Summing over $i$ and using the triangle inequality we obtain (2.4).

In the case that the partitioning is equi-distance, we have the following corollary: 
Corollary 2.4. With the assumptions in Theorem 2.3 we have

$$
\left|\int_{a}^{b} f(x) d x-T\left(f, I_{n}\right)\right| \leq \frac{M-m}{8 n}(b-a)^{2},
$$

where $I_{n}$ denotes the partitioning defined in (2.2).

We comment that $M-m<2\left\|f^{\prime}\right\|_{\infty}$ since $m<M$. So,(2.4) and (2.5) are sharper error estimates than (2.1) and (2.3)respectively. Using 'Theorem 2.5 we have the following corollary:

Corollary 2.8. Given any $\varepsilon>0$, if

$$
n \geq\left[\frac{(M-m)(b-a)^{2}}{8 \varepsilon}\right]+1
$$

then we have

$$
\left|\int_{a}^{b} f(x) d x-T\left(f, I_{n}\right)\right| \leq \varepsilon .
$$

\section{Some comments}

If the mapping $f:[a, b] \mapsto \mathbb{R}$ is twice differentiable on $(a, b)$ and $\left\|f^{\prime \prime}\right\|_{\infty}:=\sup _{x \in(a, b)}$ $\left|f^{\prime \prime}(x)\right|<\infty$, the trapezoidal quadrature rule satisfies the following classical second-order estimate:

$$
\left|\int_{a}^{b} f(x) d x-T\left(f, I_{h}\right)\right| \leq \frac{\left\|f^{\prime \prime}\right\|_{\infty}}{12} \sum_{i=0}^{n-1} h_{i}^{3},
$$

where $I_{h}, T$ and $h_{i}$ are as defined in Theorem 2.1. Although our estimates in the previous section are of first order accuracy, they depend only on $\left\|f^{\prime}\right\|_{\infty}$. In many practical situations, integrands are not continuously twice differentiable so that (3.1) can not be used. Thus, our results here provide some first order estimates for the trapezoidal rules. One simple example to demonstrate this is $f(x)=(x-a)^{P}$ with $1<p<2$. In this case $f^{\prime \prime}(x)$ is not bounded $(a, b)$ but $f^{\prime}(x)$ is continuous on $[a, b]$. So, (3.1) does not apply to this example and the results in the previous section can be used to estimate the error in the numerical integration of this $f$ by the trapezoidal rule.

\section{References}

(1) R. P. Agarwal and S. S. Dragomir, "An application of Hayashi's incquality for differentiable functions," Computers Math. Appl., 32(6)(1996), 95-99.

[2] D. S. Mitrinvoić, J. E. Pcčarić and A. M. Fink, Classical and New Inequalities in Analysis, Kluwer Academic Publishers, Dordrecht-Boston-London, 1993.

[3] D. S. Mitrinović, J. E. Pečarić and A. M. Fink, Incqualitics for functions and their intcgrals and derivatives, Kluwer Academic Publishers, Dordrecht-Boston-London, 1994.

Department of Applied Mathematics, the University of Transkey, South Africa School of Mathematics and Statistics Curtin University of Technology, GPO Box U1987, Perth
6001 , Australia 